\title{
Inquiétants acousmates
}

La diablerie d'Ansacq [1730]

Jean-Marie Privat

\section{(2) OpenEdition}

Journals

Édition électronique

URL : https://journals.openedition.org/clo/8723

DOI : $10.4000 /$ clo.8723

ISSN : 2266-1816

Éditeur

INALCO

Édition imprimée

Date de publication : 30 juin 2020

Pagination : 219-224

ISBN : 978-2-85831-392-1

ISSN : 0396-891X

Référence électronique

Jean-Marie Privat, "Inquiétants acousmates », Cahiers de littérature orale [En ligne], 87 | 2020, mis en ligne le 16 septembre 2021, consulté le 09 novembre 2022. URL : http://journals.openedition.org/clo/ 8723 ; DOI : https://doi.org/10.4000/clo.8723

\section{(c) (i) (8)}

Creative Commons - Attribution - Pas d'Utilisation Commerciale 4.0 International - CC BY-NC 4.0 https://creativecommons.org/licenses/by-nc/4.0/ 


\title{
Inquiétants acousmates La diablerie d'Ansacq [1730]
}

\author{
Jean-Marie Privat \\ Université de Lorraine, CREM
}

Le Mercure de France de décembre 1730 propose à la sagacité de ses lecteurs la « Relation d'un phénomène très extraordinaire ${ }^{1}{ }$. Simon Treüillot de Ptoncour, docteur en théologie et curé d'Ansacq [en Beauvaisis], y présente en effet avec force détails une véritable enquête sur un «prodige » nocturne qui se serait déroulé - aux dires de ses ouailles - dans la proche campagne et jusque dans leur village, une nuit de janvier de la même année ${ }^{2}$. Il s'agit d'une violente, composite et incompréhensible manifestation sonore qui effraie réellement les villageois et qui trouble profondément leur prêtre, partagé entre scepticisme philosophique de principe et inquiétude religieuse de clerc. C'est d'ailleurs cette troublante incertitude intellectuelle sur les causes - physiques - ou mieux sur les origines - magico-religieuses - de ces affolantes cacophonies aériennes qui le conduisent à présenter son dilemme au discernement de la petite communauté savante du Mercure, comme d'usage à l'époque. Il soumet donc le cas « afin que des personnes plus éclairées que moi [lui] puissent exercer leurs esprits et leurs

1. Mercure de France, décembre 1730, second volume, Paris, p. 2804-2833. « Source gallica.bnf.fr/BnF »

2. L'événement « prodigieux » et sa cohorte de bruits « épouvantables » se répétera la nuit du 24 mai de la même année 1730 ainsi qu'à la veille de la Toussaint et de la fête des morts. 
pénétrations à chercher les causes naturelles ou surnaturelles d'un évènement si extraordinaire » (Mercure de France, 1730, p. 2811).

C'est ainsi que notre savant curé de village commence par résumer son propos doublé d'entrée par une proposition lexicologique qui fera date ${ }^{3}$.

Il s'agit d'un bruit extraordinaire dans
l'air, qui a toutes les apparences d'un Pro-
dige; prefque tous les habitans du lien ò
il s'eft fait, affurent, jurent do proteftent l'a-
voir bien entendu.
Ces Témoins, comme gens de la Campa-
gne, appellent cet évenemsnt un Sabbat; les
efprits forts l'appelleront comme ils voudront,
¿ pourront raifonner, ou plutôt badiner à
leur aife.
Pour moi, dans la Differtation que jay
mife à la fin, je lui donne le nom grec d'A-
koufmate, pour flgnifier une chofe extraor-
dinaire qui s'entend dans l'air, comme on
donne le nom grec de Phénomene, aux
chofes qui paroiffent extraordinairement dans
le même Element; mais je me garde bien de
rien décider fur le fond ni fur les caufes.

Laissons-lui encore la parole - la plume d'oie - pour situer plus précisément son propos et entendre son émoi aussi.

3. Dès son édition de 1752, le fameux Dictionnaire de Trévoux donne la définition suivante du terme «Acousmate » ou « Akousmate » : «Physique. Terme nouvellement inventé pour exprimer un phénomène qui fait entendre en l'air un grand bruit semblable à celui de plusieurs voix humaines et de différents instruments, ce que l'on assure être arrivé au village d'Ansacq, près Clermont-en-Beauvaisis en 1730. » 
RELAT IO N d'un bruit extraordinairs comme de voix bumaines, entendu dans l'Air par plufteurs Particuliers de la $\mathrm{Pa}$ roifse d'Anfacq, Diocéfe de Beauvais, la nuit du 27. au 28. fanvier 1730.

T E Samedi 28. Janvier de la prefente L année, le bruit fe répandit dans la Paroiffe d'Anfacq, près Clermont en Beauvoifis, que la nuit précédente plufieurs Particuliers des deux fexes, avoiene entendu dans l'Air une multitude prodigieufe comme de voix humaines de differens tons, groffeurs \& éclats, de tous âge, de tout fexe, parlant \& criant toutes enfemble, fans néanmoins que ces ParI I.Y ol. ticu-

2808 MERCURE DE FRANCE ticuliers ayent pu rien diftinguer de ce que les voix articuloient; que parmi cetre confufion de voix, on en avoit recontu \& diftingué un nombre infini qui pouffoie des cris lugubres \& lamentables, comme de perfonnes affligées, d'autres des cris de joye \& des ris éclatans, comme de perfonnes qui fe divertiffent; quelques-uns ajoûtent qu'ils ont clairement diftingué parmi ces voix humaines, foit-difant, les fons de differens inftumens.

La plupart des témoins de auditu de ce « charivari épouvantable 》 (Mercure, 1730, p. 2818) en cette nuit du 27 au 28 janvier 1730 étaient dans leurs lits ; quelques rares autres s'en allaient nuitamment à la ville pour leurs commerces ou 
regagnaient leurs maisons (Mercure, 1730, p. 2812). Le curé du village mène alors une sorte d'enquête auprès de ses meilleurs paroissiens et enregistre par écrit leurs dépositions, signées. Voici un extrait de ce verbatim :

Ils [les frères Descoulleurs, laboureurs] seraient arrivés environ deux heures après minuit au-dessus des murs du Parc d'Ansacq [...] et que prêts à descendre par un sentier qui côtoie ces murs, s'entretenant de leurs affaires, ils auraient été tout à coup interrompus par une voix terrible, qui leur paraissait éloignée d'eux d'environ vingt pas ; qu'une autre voix semblable à la première aurait répondu sur le champ du fond d'une gorge entre deux montagnes, à l'autre extrémité du village, et qu'immédiatement après, une confusion d'autres voix se seraient fait entendre [...], articulant certain jargon glapissant $[. .$.$] , comme$ s'il y eût trois ou quatre cent personnes [...]. Et parmi tout cela les sons de différents instruments. (Mercure, 1730, p. 2812-2813)

Les dépositions se succèdent et toutes évoquent le même tumulte effrayant de voix anonymes criardes et cette même musique de tous les diables, ce même tohu-bohu de mauvais augure :

Ce 24 mai 1730 a comparu [sic] Nicolas Delaplace, laboureur, âgé d'environ 45 ans, demeurant audit Ansacq, a déclaré [...] qu'il aurait ouï comme un nombre prodigieux de personnes de tout âge et de tout sexe qui passait devant sa maison sise proche de l'église et au milieu du village, formant un bruit confus mais éclatants de voix comme humaines, mêlées de différents instruments ; qu'alors la crainte $[. .$.$] l'aurait obligé de refermer promptement sa porte et$ de se remettre au lit d'où il aurait encore entendu le même bruits et les mêmes voix, comme si elles eussent monté la rue et passé devant la maison presbytérale. (Mercure, 1730, p. 2820-2821)

Ces oralités agressives et confuses, ces ténébreuses et inouïes dissonances sonores attestées par une bonne vingtaine de témoins interrogés séparément ébranlent les certitudes du docteur en théologie, lui qui dans un premier temps n'ajoutait guère foi à ces « sortes de bruits populaires »(Mercure, 1730, p. 2808):

Cette nouvelle vint bientôt jusqu'à moi [...]. Je me contentai écrit-il dans sa Relation - d'abord de rire et de la regarder [cette vox populi] comme un effet ordinaire d'une imagination blessée et frappée de la frayeur qu'inspirent ordinairement les ténèbres 
de la nuit, surtout à des esprits grossiers et ignorants comme ceux de la plupart des gens de la campagne qui ajoutent plus de foi aux contes nocturnes [...] et ridicules [...] qu'aux vérités essentielles de l'Évangile et de la Religion. (Mercure, 1730, p. 2808)

C'est ainsi que le docte curé d'Ansacq - prenant acte de tous ces témoignages convergents et en quelque façon crédibles... - rédige pour l'entendement de ses lecteurs une description topographique du village et de ses environs immédiats. Il lui importe en effet d'expliquer - voire de s'expliquer... - pourquoi l'explication purement acoustique ne saurait rendre compte des phénomènes hétérophoniques perçus par tous, lui excepté. On n'entend guère que ce que l'on attend ${ }^{4} .$. Treüillot voudrait toutefois vérifier expérimentalement - in situ - que l'hypothèse d'une illusion de l'ouïe - «si sujette à l'erreur » - est à écarter.

4. La culture théologique et les responsabilités pastorales du curé ne le prédisposent pas à entendre l'ensauvagement oral et nocturne du monde qui épouvante les villageois de la paroisse, eux-mêmes semblant ne plus être en position d'interpréter l'inquiétante et insistante étrangeté de ces obscures processions nocturnes, avatar culturel insu sans doute - ou devenu indicible... - des bruyants cortèges aériens et malveillants des mauvais morts [enfants morts sans baptêmes, liaisons incestueuses, malheurs à répétition, bref désordres biologiques ou autres transgressions rituelles]. Une cosmologie perdue et éperdue... Un imaginaire [la créance culturelle au retour des morts] qui se diluerait dans l'imagination [de paysans incultes] ? En 1730, le retour cyclique d'une cohorte agressive de morts chez les vivants est en effet une hétérodoxie particulièrement impensable en bonne théologie chrétienne post-tridentine. Une sottise de ce genre, tout au plus ? C'est le « Je sais bien, mais quand même » du curé Treüillot. Et les villageois pour leur part sont moins dépossédés de la mémoire collective et orale de récits de revenants qui prolifèrent toujours sous une forme ou sous une autre que de l'affiliation explicite et assumée au très ancien folklore européen dit de la chasse sauvage ou mesnie Hellequin et plus encore peut-être au sens contre-rituel de son charivari diabolique. C'est parce qu'on est catholique à Ansacq qu'on en réfère au prêtre pour en quelque façon entendre la signification de ces bruits et de ces voix de l'au-delà voire pour conjurer quelque malheur qui plane pour d'obscures ou inavouables raisons sur la petite communauté. Un témoin des plus fiables ne conclut-il pas sa déclaration en assurant que « si sa déposition était défectueuse, c'était plutôt pour n'avoir pas tout dit que pour avoir amplifié »? (Mercure, 1730, p. 2815). Le symptôme est là, mais le saint homme est hésitant.

Nous préparons un ouvrage d'anthropologie culturelle et historique de ces conflits de cosmologies qui sont autant de systèmes symboliques eux-mêmes hétérogènes et en crise. Notre hypothèse est que cette Relation est révélatrice d'un double malaise civilisationnel ; malaise dans la culture des élites de province qui continuent à s'interroger tant bien que mal sur l'ordre physique et/ou le désordre cosmique ; malaise aussi dans la culture d'un peuple des campagnes, certes christianisé, mais qui hérite d'une mémoire culturelle en lambeaux mâtinée de pensée magique. L'un et l'autre à l'aurore du siècle des Lumières. 


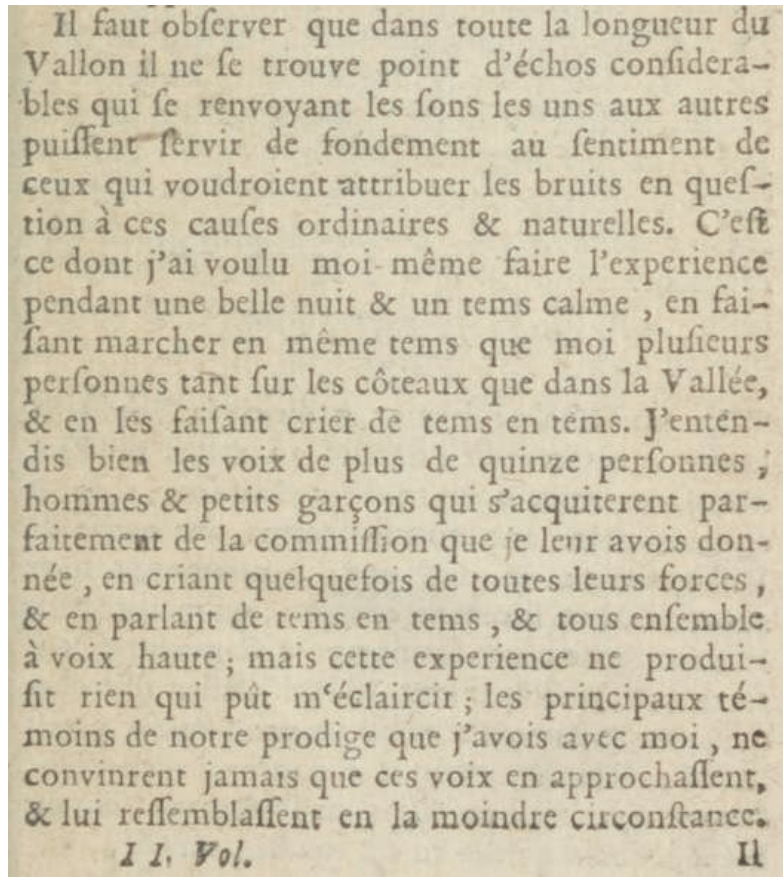

Tel est le fruit de son expérience : « une plus grande incertitude » (Mercure, 1730, p. 2832). C'est ainsi que pour désigner - c'est son mot - sinon pour rendre raison de ces bruits étonnants et détonants il propose à la communauté lettrée le mot akousmate. Le terme akousmate (écrit à la grecque par Treüillot) signifiera donc « une chose qui se fait entendre extraordinairement » (Mercure, 1730, p. 2825). C'est en effet l'oralité de cette chose qui nous intéresse ici du point de vue acoustémologique ou acoulogique, pour autant que l'inquiétante performance acousmatique est bien ce maelstrom sonore et sans corps dont - à la différence de l'écho et de ses familières illusions - on ne saurait ni percevoir les causes réelles ni entendre les origines probables $5 . .$.

Ni vu ni (re-)connu. Entendu.

5. Pour entendre d'une autre oreille épistémologique ces phénomènes, on se reportera particulièrement bien sûr à Schaeffer Pierre, 1966, «L'acousmatique » in Traité des objets musicaux, Seuil, Paris, p. 91-99. Voir aussi les travaux d'acoulogie de Michel Chion et pour une première initiation aux acousmates et autres fantômes sensoriels pour parler comme Merleau-Ponty, se reporter à son Glossaire : http://www.lampe-tempete.fr/ ChionGlossaire.html. 